\title{
Desarrollo de un instrumento para la evaluación del malestar emocional para pacientes renales en diálisis
}

\author{
Helena García-Llana ${ }^{1}$, Rocío Rodríguez-Rey², María Jesús Rollán de la Sota ${ }^{3}$, Fabiola Dapena Vielba ${ }^{4}$, Fernando \\ Ramos Peña ${ }^{5}$, Filo Trocoli González ${ }^{1}$, Rafael Selgas Gutiérrez ${ }^{1}$ \\ ${ }^{1}$ Hospital Universitario La Paz-IdiPAZ. Madrid. ${ }^{2}$ Facultad de Ciencias de la Salud. Departamento de Psicología. \\ Universidad Internacional Isabel I de Castilla. Madrid. ${ }^{3} \mathrm{Hospital}$ Universitario Clínico de Valladolid. ${ }^{4}$ Consorci \\ Sanitari El Garraf de Barcelona. ${ }^{5}$ Hospital Universitario Cruces de Bilbao. España
}

\section{Resumen}

Objetivo: Actualmente no existen herramientas validadas para evaluar el malestar emocional en pacientes con enfermedad renal crónica, pese a su alta prevalencia en todas las etapas de la patología. Este trabajo describe el desarrollo de un instrumento breve semiestructurado para la Evaluación del malestar Emocional de los pacientes renales en Diálisis (EE-D).

Método: EI EE-D incluye cinco preguntas con distintos formatos de respuesta (dicotómico, Likert y preguntas abiertas) para evaluar tristeza, ansiedad, preocupaciones, recursos para afrontar la enfermedad, signos externos de malestar y observaciones. Los ítems de la primera versión se sometieron a un estudio interjueces $(N=10)$. Posteriormente se realizó un estudio piloto multicéntrico ( $N=25$ pacientes en 5 hospitales).

Resultados: Tras el estudio interjueces un ítem fue eliminado, y otro añadido. Tras el piloto no hubo modificaciones. La puntuación media (sobre 10) fue 4,16 (DT: 3,41$)$ en tristeza y 3,68 (DT: 3,48$)$ en ansiedad. El $48 \%$ refirió preocupaciones familiares, el $44 \%$ preocupaciones sobre su estado emocional-psicológico y el $40 \%$ sobre su enfermedad/tratamientos. Los principales recursos identificados fueron la esperanza en la posibilidad de trasplante y el apoyo social. A juicio de enfermería el $24 \%$ presentan expresión facial de malestar y el $16 \%$ otros signos de malestar.

Correspondencia:

Helena García-Llana

Servicio de Nefrología. Hospital General

Hospital Universitario La Paz. IdiPAZ.

Paseo de la Castellana 261. 28046 Madrid. España

E-mail: helenagllana@hotmail.com
Discusión: El EE-D muestra unas garantías psicométricas preliminares. Ha sido distribuido en las Unidades de Diálisis de todo el territorio nacional con el objetivo de su implementación clínica en un marco investigador, lo que permitirá el desarrollo de futuros estudios de validación multicéntricos.

PALABRAS CLAVE

- MALESTAR EMOCIONAL

- EVALUACIÓN

- ENFERMEDAD RENAL CRÓNICA

- DIÁLISIS

- ENFERMERÍA

Development of a Questionnaire to Assess Emotional Distress in Renal Patients undergoing Dialysis

\begin{abstract}
Objective: Currently there are no validated assessment tools to explore emotional distress in patients with chronic kidney disease, even though it is very prevalent in all the phases of the disease. This study describes the development of a brief semi-structured interview to assess emotional distress in renal patients undergoing Dialysis (EE-D).

Method: The EE-D includes five questions with different response formats (dichotomized, Likert scales and open-questions) to assess sadness, anxiety, concerns, and resources to cope with illness, external signs of distress and other considerations. An interjudge process was used with the items of the first version of the EE-D ( $N=10$ experts). Hereafter a pilot multicentric study was carried on ( $N=25$ patients in 5 hospitals).
\end{abstract}


Results: After the interjudge analysis one item was removed from the EE-D, and one item was added. After the pilot study there were no changes in the tool. The average score (range 0 to 10 ) in sadness was 4.16 $(S D=3.41)$ and $3.68(S D=3.48)$ in depression. $A$ total of $48 \%$ of the participants reported family concerns, $44 \%$ concerns about their psychological/ emotional state and $40 \%$ about their illness/treatments. The most important resources to cope with the illness were their hope in the possibility of a transplantation and their social support. According to the nursing staff who conducted the interviews $24 \%$ of the patients showed facial expression of distress and $16 \%$ other external signs of distress.

Discussion: The EE-D has shown adequate preliminary psychometric properties. It has been recently distributed to all the dialysis units in Spain with the objective of conducting a multicentric validation study.

\section{KEYWORDS}

- EMOTIONAL DISTRESS

- ASSESSMENT

- CHRONIC KIDNEY DISEASE

- DIALYSIS

- NURSING

\section{Introducción}

La Enfermedad Renal Crónica (ERC) es la patología que se produce por la pérdida mantenida o crónica de las funciones vitales que desempeñan los riñones. El proceso de la ERC cursa a través de 5 estadios. La progresión entre estadios se realiza en función de la gravedad del fallo renal, siendo la situación más leve el estadio 1 , y la más grave el $5^{1}$. Cuando el paciente se encuentra en los estadios 4 y 5 decimos que tiene una ERC avanzada (ERCA $)^{2}$. El Estudio Epidemiológico de la Insuficiencia Renal Crónica en España, EPIRCE, realizado por la Sociedad Española de Nefrología ${ }^{3}$ refiere que la prevalencia de la insuficiencia renal crónica en estadios 3-5 es del 6,8\% entre la población española, siendo de un 3,3\% entre los 40 y los 64 años, y de un $21,4 \%$ en mayores de 64 años. En la situación de ERCA, los pacientes son subsidiarios de tratamiento renal sustitutivo (TRS) 0 manejo renal conservador. Existen tres modalidades de TRS: hemodiálisis, diálisis peritoneal y trasplante de riñón.
Durante el proceso de la enfermedad renal el paciente se enfrenta a múltiples dificultades y estresores, como los efectos secundarios de la diálisis (ej. prurito, calambres), las múltiples pautas nutricionales y de restricción hídrica, problemas en su relación con su entorno social (ej. alta dependencia) o dificultades laborales entre otros ${ }^{4,5}$. Todo ello conduce a un alto riesgo de sufrir problemas psicológicos, destacándose una alta prevalencia de depresión y de ansiedad ${ }^{6}$. La tasa de depresión en distintos estudios oscila entre el 35 y el $44 \%{ }^{7,8}$. Con respecto a los trastornos de ansiedad, se ha encontrado una prevalencia del $45,7 \%$ en pacientes en hemodiálisis?.

Dada la complejidad que supone la ERC en sus estadios avanzados, existe una alta prevalencia de alteraciones emocionales en la población de pacientes renales en diálisis. Aliviar el sufrimiento de los pacientes es uno de los objetivos prioritarios de la medicina del siglo $\mathrm{XXI}^{10}$. Para poder realizar esta labor es necesario incorporar a los cuidados rutinarios una evaluación del estado emocional del paciente, lo que podría favorecer que este problema se detectara y tratase adecuadamente, ayudando a mejorar el bienestar psicológico y la calidad de vida ${ }^{11}$. Actualmente no existe ningún instrumento de screening específicamente desarrollado para evaluar el estado emocional de los pacientes con ERC en tratamiento de diálisis. Por ello, nos proponemos diseñar un instrumento breve y de fácil aplicación que permita una detección temprana del posible malestar emocional de los pacientes sometidos tanto a hemodiálisis como a diálisis peritoneal. Debido a que los profesionales de enfermería son los que más contacto mantienen con los pacientes renales, y a la intensidad de los vínculos enfermero/a-paciente que suelen establecerse ${ }^{12}$ pensamos que son los profesionales idóneos para realizar este trabajo de detección preliminar.

En el contexto de otras enfermedades, sí se han desarrollado y validado herramientas que permiten valorar el estado emocional de los pacientes. La herramienta de Detección de Malestar Emocional de Maté et al. ${ }^{13}$, desarrollada en el ámbito de los cuidados paliativos, ha demostrado tener adecuadas propiedades psicométricas y ser de utilidad para explorar el malestar emocional de los pacientes con enfermedades oncológicas avanzadas ${ }^{14}$. Esta herramienta, a su vez, se basó en las escalas de Bruera et al. ${ }^{15}$, Chochinov et al. ${ }^{16}$, Comas et al. ${ }^{17}$, Holland et al. ${ }^{18}$ y Hürny et al. ${ }^{19}$. Incluye cinco preguntas para evaluar malestar general, preocupaciones de distintos tipos (ej. económicas, familiares), esfuerzo perci- 
bido de hacer frente a la situación y signos externos de malestar ${ }^{13}$.

Para desarrollar la escala de evaluación del estado emocional para pacientes en diálisis, complementamos la herramienta de Detección de Malestar Emocional con el Modelo de Potenciación ${ }^{20-22}$, y el modelo de sufrimiento de Bayés et al. ${ }^{23}$. Según estos modelos, la adaptación y la experiencia de sufrimiento no dependen únicamente de las amenazas (físicas 0 psicosociales) a las que se enfrente el paciente, sino también de los recursos o factores de protección ${ }^{24}$ que las personas perciben que tienen para hacer frente a dicha amenaza ${ }^{20-24}$. Ambos elementos, amenazas y recursos, son necesarios para hablar de sufrimiento. De este modo, por amenazadora que sea una situación, si la persona dispone de recursos para hacerle frente y confía en su capacidad para ponerlos en marcha no existirá sufrimiento. Basándonos en esta perspectiva, la herramienta que vamos a desarrollar en este trabajo incluirá no sólo amenazas, sino también recursos percibidos por el paciente para hacerles frente.

En resumen, el objetivo de este trabajo es el desarrollo de un instrumento breve que permita al personal de enfermería nefrológica evaluar el estado emocional de los pacientes renales en diálisis. Esperamos que este instrumento sea de utilidad para facilitar la detección del malestar emocional en estos pacientes con el fin de proporcionarles un apoyo emocional más específico, así como facilitar la derivación a otros profesionales (trabajador social, psicólogo, etc.) que pudiesen ofrecerles una atención más especializada.

\section{Método}

\section{a) Desarrollo del instrumento}

Para desarrollar la herramienta de evaluación del Estado Emocional en pacientes en Diálisis (EE-D) los autores nos basamos en la herramienta de Detección de Malestar Emocional de Maté et al. ${ }^{13,14}$, que complementamos desde el marco del modelo de potenciación ${ }^{20-22}$, y el modelo de sufrimiento de Bayés et al. ${ }^{23}$, de manera que también se incluyesen recursos percibidos por el paciente.

La primera versión de la herramienta desarrollada incluyó las siguientes áreas:

1) Una valoración breve de su estado de ánimo (tristeza y ansiedad).
2) Las amenazas a las que se tienen que enfrentar los pacientes.

3) Sus recursos percibidos para hacerles frente.

4) Evaluación de signos externos de malestar, percibidos por el personal de enfermería.

El instrumento preliminar constaba de cinco preguntas. En la primera de ellas, con una escala de 0 a 10 se pregunta acerca de la tristeza y el nerviosismo que está experimentando el paciente. En esta primera pregunta nuestro objetivo es detectar la presencia de posible ansiedad y/o depresión. En la segunda pregunta se exploran posibles áreas de preocupación (familiar, etc.), con el fin de conocer las preocupaciones del paciente detectar si es posible ofrecerle alguna ayuda específica. La tercera y cuarta preguntas exploran recursos del paciente para hacer frente a la enfermedad, y la quinta explora signos externos de malestar. Por último se incluye un último apartado llamado "observaciones" donde el profesional puede incluir comentarios breves.

\section{b) Estudio interjueces}

El diseño preliminar del instrumento se sometió a un análisis interjueces para verificar la validez de contenido y la validez aparente (face validity). Para ello, esta primera versión se presentó a un grupo interdisciplinar de diez expertos, de los cuales seis eran enfermeras con amplia experiencia en diálisis, dos eran pacientes en programa de hemodiálisis crónica, uno era nefrólogo coordinador del área de diálisis de un hospital terciario y uno era psicólogo clínico.

En análisis interjueces se llevó a cabo en dos fases. En la primera se les pedía que emitieran un juicio en cuanto al grado en que cada ítem era relevante o comprensible, con respecto a si sugerían alguna mejora con respecto al mismo para hacerlo más comprensible o relevante y con respecto a si sugerían su eliminación. En la segunda fase se les preguntaba si añadirían algún ítem que no estuviese previamente considerado. Por último se les preguntaba si tenían algún comentario adicional acerca del instrumento que quisieran añadir. El criterio establecido para reformular los ítems se baso en el porcentaje de acuerdo entre jueces expertos respecto a la valoración de la comprensión y la relevancia del ítem. Asimismo se les preguntaba si harían alguna sugerencia para mejorar el ítem, o si lo eliminarían del instrumento.

${ }^{1}$ Se obtuvo permiso de los autores del instrumento de Detección de Malestar Emocional para adaptar su herramienta al ámbito de la enfermedad renal crónica avanzada. 
Para la toma de decisiones acerca del mantenimiento o eliminación de los ítems nos basamos en los criterios empleados en Remor \& The Hemophilia QoL. ${ }^{25}$ para la construcción de un cuestionario de calidad de vida para pacientes con hemofilia. Siguiendo estos criterios, los ítems eran reformulados si: a) un $20 \%$ de los jueces 0 más consideraba que no eran relevantes, o b) un $20 \%$ o más de los jueces consideraba que no eran comprensibles o si c) al menos un $20 \%$ de los jueces sugieren su modificación. Los ítems eran eliminados si un $20 \%$ de los jueces o más lo sugerían, y se añadían nuevos ítems si había alguna sugerencia en común por parte de al menos el $20 \%$ de los jueces. Todas las valoraciones las realizaron en un formulario estandarizado diseñado para tal fin.

\section{c) Estudio piloto}

Empleando la versión del instrumento obtenida tras el análisis interjueces, se realizó un estudio piloto multicéntrico con el objetivo fue evaluar posibles dificultades 0 aspectos mejorables en la comprensión, relevancia o formulación de las preguntas.

La muestra estuvo compuesta por 25 pacientes que recibían tratamiento de diálisis en alguno de los siguientes centros:

- Hospital Universitario Clínico de Valladolid ( $N=$ 6).

- Hospital Universitario Río Hortega de Valladolid $(\mathrm{N}=5)$.

- Hospital Universitario Cruces de Bilbao $(\mathrm{N}=5)$.

- Unidad Diálisis de Vilanova en Barcelona $(\mathrm{N}=5)$.

- Hospital Universitario La Paz de Madrid $(\mathrm{N}=4)$.

Los pacientes cumplían los siguientes criterios de inclusión: a) Ser mayor de 18 años; b) Estar diagnosticado de ERCA; c) Estar recibiendo tratamiento de diálisis; d) Tener una adecuada comprensión del idioma que no dificulte la aplicación del instrumento y e) Dar su consentimiento verbal para ser evaluado.

Los criterios de exclusión fueron: a) Llevar menos de tres meses en el programa actual de diálisis; b) Presentar deterioro cognitivo y c) Padecer alguna enfermedad psiquiátrica activa que impida la correcta comprensión y respuesta de la entrevista o altere el estado de conciencia.

Para la realización de las entrevistas se realizó una planilla de evaluación que, con respecto a cada ítem incluía si el paciente lo comprendía o no, si lo consideraba relevante o no, y si ofrecía alguna sugerencia de mejora. El entrevistador anotaba las respuestas del paciente de cara a detectar información solapada con respecto a otras preguntas del instrumento y la adecuación de las preguntas La duración media de la entrevista fue de 11,16 minutos (DT: 4,17). Las entrevistas se Ilevaron a cabo en las Unidades de Hemodiálisis y Diálisis Peritoneal. Para la toma de decisiones acerca del mantenimiento o eliminación de los ítems nos volvimos a basar en los criterios empleados en Remor \& The Hemophilia QoL. ${ }^{25}$ para la construcción de un cuestionario de calidad de vida para pacientes con hemofilia.

\section{Resultados}

\section{a) Estudio interjueces}

\section{Análisis descriptivos de los jueces}

Ocho de los jueces son mujeres $(80 \%)$, con una edad media de 47 años (DT: 14,21). La media de años de experiencia en el área de diálisis del grupo es 18 (DT: 10,47 ) ya fuera como paciente o como profesional sanitario.

\section{Funcionamiento de los ítems}

Los resultados con respecto a la modificación y la eliminación de los ítems se reflejan en la Tabla 1.

Únicamente el ítem $2 \mathrm{~b}$, posibles preocupaciones sobre el área económica, es eliminado del cuestionario debido a que un $30 \%$ de los jueces sugiere su eliminación. No se realizan modificaciones en ninguno de los ítems.

Con respecto a las sugerencias de adición de ítems, se realizan un total de tres, tal y como se refleja en la Tabla 2. 
[ Helena García-LLana ]

Desarrollo de un instrumento para la evaluación del malestar emocional para pacientes renales en diálisis

Tabla 1. Tasa de respuesta de la evaluación a 10 jueces sobre los ítems del instrumento.

\begin{tabular}{|c|c|c|c|c|c|}
\hline Ítem & Comprensión (\%) & Relevancia (\%) & $\begin{array}{c}\text { Sugerencia } \\
\text { Modificación } \\
(\%)\end{array}$ & $\begin{array}{c}\text { Sugerencia } \\
\text { eliminación } \\
(\%)\end{array}$ & Decisiór \\
\hline la Tristeza & 100 & 100 & 0 & 0 & * \\
\hline lb Ansiedad & 100 & 100 & 0 & 0 & * \\
\hline 2a Preocupaciones familiares & 90 & 100 & 10 & 0 & * \\
\hline 2b Preocupaciones económicas & 70 & 90 & 10 & 30 & Eliminar \\
\hline 2c Preocupaciones emocionales y/o psicológicas & 90 & 90 & 10 & 10 & * \\
\hline 2d Preocupaciones espirituales y/o religiosas & 90 & 90 & 10 & 10 & * \\
\hline 2e Preocupaciones enfermedad y/o tratamientos & 90 & 100 & 10 & 10 & * \\
\hline $2 f$ Preocupaciones relación profesionales sanitarios & 90 & 100 & 10 & 10 & * \\
\hline 2g Otras preocupaciones & 90 & 100 & 10 & 10 & * \\
\hline 3 Qué le ayuda & 90 & 90 & 10 & 10 & * \\
\hline 4 Qué le hace ilusión o pone contento & 90 & 90 & 10 & 10 & * \\
\hline 5a Expresión facial malestar & 100 & 90 & 0 & 10 & * \\
\hline 5b Aislamiento desadaptativo & 90 & 90 & 10 & 10 & * \\
\hline 5c Demanda constante de atención & 100 & 90 & 0 & 10 & * \\
\hline $\mathbf{5 d}$ Alteraciones del comportamiento en diálisis & 100 & 90 & 10 & 10 & * \\
\hline 5e Otros signos externos de malestar & 100 & 90 & 0 & 10 & * \\
\hline 6 Observaciones & 100 & 90 & 0 & 10 & * \\
\hline
\end{tabular}

Nota: * Ítems que se mantienen sin modificaciones en el cuestionario.

Tabla 2. Sugerencias de adición de ítems por parte de $\mathrm{N}=10$ jueces del estudio interjueces.

\begin{tabular}{l|c|c|}
\hline Sugerencia(s) & Frecuencia & Decisión \\
\hline $\begin{array}{l}\text { Desde que está en diálisis } \\
\text { ¿Qué cosas le fastidian realmente? }\end{array}$ & 1 & No añadido \\
\hline $\begin{array}{l}\text { Añadir el área "Laboral" } \\
\text { en el apartado 2. }\end{array}$ & 3 & Añadido \\
\hline $\begin{array}{l}\text { Añadir en signos externos "verborreico" } \\
\text { e "invasión de espacios" }\end{array}$ & 1 & No añadido \\
\hline
\end{tabular}

Nota: La frecuencia se refiere al número de personas que hacen la sugerencia que se indica.

Solo uno de los 3 ítems sugeridos es incorporado al instrumento. Dicho ítem se refiere a la inclusión del área laboral. Estudios recientes ${ }^{26}$, han demostrado el valor predictivo de la variable "ocupación laboral", de manera independiente, sobre las dimensiones físicas de la calidad de vida de los pacientes en ambas modalidades de diálisis. Concluimos por tanto que tras realizar el análisis interjueces solamente un ítem es añadido, y uno es eliminado.

\section{b) Estudio piloto}

\section{Análisis descriptivos de la muestra}

Personal de enfermería encargado de la recogida de datos:

Los 25 profesionales son mujeres con una edad media de 49,8 años (DT: 9,1) y una media de 20,4 (DT: 11,21) años de experiencia.

\section{De los pacientes:}

El $56 \%$ son hombres. El $72 \%$ están casados. Únicamente el $8 \%$ tiene ocupación laboral en el momento de la evaluación. Con respecto a la técnica de diálisis recibida, el $76 \%$ está en hemodiálisis, el 16 $\%$ en diálisis peritoneal automatizada y el $8 \%$ en diálisis peritoneal continua ambulatoria. El $24 \%$ han sido trasplantados previamente en el momento de la evaluación, y el $40 \%$ han pasado por otra técnica de diálisis previa. El $52 \%$ son candidatos a trasplante. La media del índice de comorbilidad de Charlson es de 3,45 (DT: 1.89). 


\section{[ Helena García-LLana ]}

Desarrollo de un instrumento para la evaluación del malestar emocional para pacientes renales en diálisis

\section{Funcionamiento de los ítems}

Los resultados acerca de la eliminación o modificación de los ítems en base a los criterios mencionados se encuentran recogidos en el Tabla 3. Los resulta- dos sobre los ítems sugeridos, así como la toma de decisiones sobre su incorporación o no al instrumento se encuentran en la Tabla 4. Como ambas tablas muestran, tras el estudio piloto ningún ítem fue añadido o eliminado del cuestionario.

Tabla 3. Tasa de respuesta de la evaluación piloto a 25 pacientes sobre los 5 ítems de la segunda versión del instrumento.

\begin{tabular}{|c|c|c|c|}
\hline Item & Comprensión (\%) & $\begin{array}{c}\text { Relevancia/ } \\
\text { No solapamiento (\%) }\end{array}$ & Decisión \\
\hline $\begin{array}{l}\text { 1. Ponga un círculo alrededor del número que mejor describa } \\
\text { cómo se ha sentido durante la semana pasada (entre } \\
0=" \text { nada triste" y } 10=" \text { muy triste } ; 0=" \text { nada nervioso" y } \\
10=" \text { muy nervioso"). }\end{array}$ & 100 & 100 & * \\
\hline $\begin{array}{l}\text { 2. Queremos valorar sus preocupaciones actuales para ver si } \\
\text { podemos ofrecerle alguna ayuda (área familiar, laboral, } \\
\text { emocional-psicológico, espiritual-religioso, La enfermedad, } \\
\text { tratamiento y/o relación con profesionales sanitarios, otros. }\end{array}$ & 96 & 88 & * \\
\hline $\begin{array}{l}\text { 3. Desde que está en diálisis ¿Qué cree que es lo que le ayuda o } \\
\text { que le ayudaría a sentirse mejor? }\end{array}$ & 96 & 84 & * \\
\hline $\begin{array}{l}\text { 4. Desde que está en diálisis ¿Qué cosas le hacen ilusión o le } \\
\text { "ponen contento". }\end{array}$ & 92 & 92 & * \\
\hline $\begin{array}{l}\text { 5. (Completar por el profesional) Señale si se observa alguno } \\
\text { de los siguientes signos externos de malestar (Expresión } \\
\text { facial que transmite malestar, aislamiento desadaptativo, } \\
\text { demanda constante de compañía y atención de la enferme- } \\
\text { ría, alteraciones del comportamiento en la sala de diálisis/ } \\
\text { Unidad de DP, Otros). }\end{array}$ & 92 & 88 & * \\
\hline
\end{tabular}

Notas: * Ítems que se mantienen en el cuestionario. Se reformulan los ítems si el porcentaje de comprensión es menor de $70 \%$. Se eliminan los ítems que cuya relevancia es inferior al $70 \%$.

Tabla 4. Sugerencias de modificación de los ítems por parte de $\mathrm{N}=25$ pacientes del estudio piloto.

\begin{tabular}{|c|l|c|l|}
\hline Item & Sugerencia(s) & Frecuencia & Decisión \\
\hline 3 & Definir mejor sentirse mejor y ayudar & 1 & No incorporar \\
\hline 4 & Dar opciones de contestación & 1 & No incorporar \\
\hline 4 & ¿Qué le pone triste de su enfermedad? & 1 & No incorporar \\
\hline 5 & $\begin{array}{l}\text { La demanda de atención puede ser } \\
\text { de otra forma, como atención de cariño }\end{array}$ & 1 & No incorporar \\
\hline
\end{tabular}

Notas: La frecuencia se refiere al número de personas que hacen la sugerencia que se indica.

\section{Resultados de los pacientes en las variables evaluadas}

La puntuación media de los 25 pacientes evaluados fue 4,16 (DT: 3,41) en tristeza y 3,68 (DT: 3,48) en ansiedad, ambas sobre 10 . El $48 \%$ refirió preocupaciones familiares, el $44 \%$ preocupaciones sobre su estado emocional-psicológico, el $40 \%$ sobre su enfermedad/tratamientos, el $12 \%$ sobre su trabajo, el $8 \%$ sobre aspectos espirituales y el $20 \%$ refiere otras preocupaciones.

Los principales recursos identificados (evaluados mediante las preguntas abiertas 3 y 4) fueron el apoyo social de su entorno cercano $\mathrm{y} / \mathrm{o}$ del personal sanitario (mencionado por un $76 \%$ ), la esperanza en la posibilidad del trasplante (mencionado por un $44 \%$ ), y mantenerse ocupado en actividades cotidianas y/o placenteras $(28 \%)$.

Con respecto a la presencia de signos externos de malestar, a juicio de enfermería el $24 \%$ de los pa- 
cientes evaluados presentan expresión facial de malestar, el $16 \%$ otros signos de malestar y el $4 \%$ demanda atención constante. Ninguno de los pacientes evaluados muestra alteraciones de comportamiento en la unidad, ni signos de aislamiento desadaptativo.

La Figura 1 muestra la versión final de la herramienta.

\section{Discusión}

El procedimiento interjueces y el estudio piloto realizados para conocer la validez de contenido de la herramienta EE-D han mostrado, de manera preliminar, que esta herramienta funciona adecuadamente cuando es aplicada por parte de los profesionales de enfermería a pacientes renales en diálisis. Asimismo, los escasos cambios realizados, son indicadores de que el cuestionario diseñado tiene una adecuada validez de contenido y aparente.

Este instrumento presenta una serie de ventajas que se han visto demostradas en los estudios realizados hasta el momento y presentados en este artículo. Es un instrumento de screening, y por lo tanto fácil de aplicar por parte del profesional de enfermería y de responder por parte de los pacientes en diálisis. Además, ha mostrado ser comprensible y recoger todos los aspectos de mayor relevancia para la evaluación del malestar emocional en enfermos renales. Por su facilidad en su aplicación, es posible incorporarlo en los protocolos de evaluación en las unidades de diálisis de cara a una posible monitorización del estado de ánimo. Adicionalmente, creemos que es un instrumento terapéutico en sí mismo, ya que facilita el inicio o consolidación del vínculo paciente-profesional sanitario, permitiendo que se aborden aspectos emocionales del paciente que, generalmente, no se habrían detectado por medio de la interacción cotidiana. Finalmente, consideramos que es un instrumento adecuado para facilitar la detección de los pacientes con mayor problemática emocional o psicológica que puedan requerir una atención más especializada por parte de profesionales del ámbito de la psicología de la salud.
Por otra parte, y no menos importante, estos estudios preliminares han permitido detectar las inquietudes de profesionales y pacientes en referencia al abordaje de la dimensión emocional en el paciente renal en diálisis. Dichas inquietudes se han visto especialmente reflejadas en los comentarios que añaden algunos de los jueces en la pregunta abierta final de la entrevista. El comentario más frecuente por parte de la enfermería ha sido la necesidad de formación, para saber cómo actuar en caso de detectar malestar emocional en los pacientes. Por su parte los pacientes tienden a destacar la utilidad del instrumento, así como la importancia y la adecuación que tiene el hecho de que la enfermería tenga en cuenta los aspectos emocionales como parte de la atención y los cuidados que reciben. 
Somos conscientes de que las mismas características que lo hacen adecuado para la práctica clínica en las unidades de diálisis, conducen a que sus características desde el punto de vista psicométrico sea limitada, por ello es especialmente importante conocer cómo funciona esta herramienta al ser aplicada a una muestra amplia de pacientes. En consecuencia, el siguiente paso de este proyecto consistirá en la realización de un estudio de validación multicéntrico con una muestra de al menos 200 pacientes lo que permitirá conocer sus propiedades psicométricas.

Por tanto, pese a no tener aún resultados concluyentes acerca de las propiedades psicométricas y el funcionamiento de esta herramienta, pensamos que esta herramienta supone un paso importante en la mejora de la atención integral de los pacientes renales en diálisis.

Por último, es necesario destacar que la utilización de esta herramienta de screening, aunque útil, no garantiza por sí sola que el personal de enfermería vaya a ser capaz de detectar el estado emocional del paciente renal y tomar las decisiones oportunas. Para ello es necesario que los profesionales que vayan a aplicarla reciban formación en estrategias de Counselling, para que se garantice un adecuado abordaje y derivación ante el malestar y el sufrimiento que puedan detectar en los pacientes.

Los autores declaran que no hay ningún conflicto de intereses.

Agradecimientos: Los autores quieren agradecer a la Sociedad Española de Enfermería Nefrológica (SEDEN) su confianza y patrocinio, elementos fundamentales en el desarrollo de esta herramienta. Así como al grupo para el estudio del estado emocional de la SEDEN** por la continuidad en el desarrollo de esta herramienta. Por último, no queremos olvidar a Laboratorios AMGEN su apoyo en la maquetación y distribución de la misma.

* María Paz Ruiz Álvarez (Hospital Universitario La Paz de Madrid), Ana Rico, María Jesús Fernández Manjón, Francisco L. Sánchez Izquierdo (Fundación Asyter), Alberto Sánchez Martín (Fundación FRIAT Castilla y León), Encarna Sánchez Meca (Fundación Hospital de Alcorcón de Madrid), Ana María Matamala Gascón (Fundación Puigvert de Barcelona), Miren Santana Díez (Hospital de Cruces de Bilbao), Alicia Gómez Gómez, Laura Baena Ruiz (Hospital del Henares de Madrid), Sonia Hermida Cerro (Hospital Infanta Cristina de Madrid), Miguel Angel Moral Caballero, $\mathrm{M}^{\mathrm{a}}$ Victoria Miranda Camarero (Hospital Universitario La
Princesa de Madrid), Alicia Quintano Pintado, Ana Dorado (Hospital Universitario Río Hortega de Valladolid), María Ángeles Martínez Terceño (Hospital Universitario Severo Ochoa de Madrid), Ana Rico González (Asyter La Mancha), Fabiola Dapena Vielba (Consorci Sanitari Garraf), Ma Jesús Rollán de la Sota (Hospital C.U. de Valladolid), y Yenyra Ravelo Valiente (Hospital del Tajo. Getafe. Madrid)

Recibido: 1 noviembre 2016

Revisado: 5 noviembre 2016

Modificado: 7 noviembre 2016

Aceptado: 15 noviembre 2016

\section{Bibliografía}

1. Soriano S. Definición y clasificación de los estadios de la enfermedad renal crónica. Prevalencia. Claves para el diagnóstico precoz. Factores de riesgo de enfermedad renal crónica. Nefrología 2004; 24 (6): 27-34.

2. Alcázar-Arroyo R, Orte-Martinez L y Otero-González A. Enfermedad Renal Crónica Avanzada. Nefrología. 2008; (Supl. 3): 3-6.

3. Otero A, de Francisco A, Gayoso P, García $F$ y EPIRCE study group. Prevalence of chronic renal disease in Spain: Results of the EPIRCE study. Nefrología. 2010; 30 (1): 78-86.

4. Cukor D, Cohen SD, Peterson RA \& Kimmel PL. Psychosocial aspects of chronic disease: ESRD as a paradigmatic illness. J Am Soc Nephrol. 2007;18:3042-3055.

5. Leiva-Santos JP, Sánchez-Hernández R, García-Llana $H$, et al. Cuidados de soporte renal y cuidados paliativos renales: Revisión y propuesta en terapia renal sustitutiva. Nefrologia. 2012;32:20-27.

6. Feroze U, Martín D, Reina- Patton A, KalantarZadeh K. \& Kopple JD. Mental health, depression, and anxiety in patients on maintenance dialysis. Iran J Kidney Dis. 2010; 4 (4): 173- 180.

7. Watnick S, Kirwin P, Mahnensmith R. \& Concato $\mathrm{J}$. The prevalence and treatment of depression among patients starting dialysis. Am J Kidney Dis. 2003; 41 (1), 105-110. 
8. Chen, C.K., Tsai, Y.C., Hsu, H.J., Wu, I.W., Sun, C.Y., Chou, C.C., et al. (2010). Depression and suicide risk in hemodialysis patients with chronic renal failure. Psychosomatics, 51, 528-528 e526.

9. Cukor D, Coplan J, Brown C, Friedman S, Newville $\mathrm{H}$, Safier, et al. Anxiety disorders in adults treated by hemodialysis: a single-center study. Am J of Kidney Dis. 2008; 52 (1): 18-136.

10. Callahan D. Death and the research imperative. N Engl J Med. 2000;324:654-6.

11. Paparrigopoulos MD, Theleritis, MD, Tzavara MS. \& Papadaki MD. Sleep disturbance in haemodialysis patients is closely related to depression. Gen Hosp Psychiat. 2009; 31: 175-177.

12. García-Llana, H. y Coca C. (2016). Cuidados Paliativos no oncológicos: Consideraciones Especiales. En: J. Barbero Gutiérrez, X. Gómez-Batiste, J. Maté Méndez y D. Mateo Ortega (Eds.). Manual para la atención psicosocial y espiritual a personas con enfermedades avanzadas: Intervención Psicológica y Espiritual. Obra Social La Caixa: Barcelona.

13. Maté J, Mateo D, Bayés, R, Bernaus $M$, Casas, $C$, González- Barboteo J, Limonero JT, López- Postigo M, Sigo A y Viel S. Elaboración y propuesta de un instrumento para la detección de malestar emocional en enfermos al final de la vida. Psicooncología. 2009; (6), Num 2-3: 507- 518.

14. Limonero JT, Mateo D, Maté-Méndez J, et al. Evaluación de las propiedades psicométricas del cuestionario de Detección de Malestar Emocional (DME) en pacientes oncológicos Assessment of the psychometric properties of the Detection of Emotional Distress Scale in cancer patients. Gaceta Sanitaria. 2012;26:145-152.

15. Bruera E, Kuehn N, Miller MJ, Selmser P, Macnillan K. The Edmonton Symptom Assessment System: a simple method for the assessment of palliative care. J Palliat Care 1991; 7: 6-9.

16. Chochinov HM, Wilson KG, Enns M, Lander S. "Are you depressed?" Screening for depression in the terminally ill. Am J Psychiatry 1997; 154 (5): 674-76.

17. Comas MD, Calafell B, Limonero JT, Bayés R. Elaboración de una escala observacional para la evaluación de la depresión en Cuidados Paliativos: estudio preliminar. Med Paliat (Madrid) 2004; 11 (2): 96-101.

18. Holland JC, Andersen B, Breitbart WS, et al. The NCCN distress management clinical practice guidelines in oncology. J Natl Compr Canc Netw. 2010;8:448-84.19

19. Hürny $C$, Bernhard J, Bacchi $M$, van Wegberg $B$, Tomamichel M, Spek U,et al. The Perceived Adjustement to Chronic Illness Scale (PACIS): A global indicator of coping for operable breast cancer parients in clinical trials. Support Care Cancer 1993; 1: 200-8.

20. Albee GW. A competency model to replace the defect model. In: Gibbs MS, Lachenmeyer JP \& Sigal J, editors. Community Psychology. Nueva York: Gardner Press; 1980 p.213-38.

21. Arranz $P$, Costa $M$, Bayés $R$, Cancio $H$, Magallón $M$, Hernández-Navarro $F$. El apoyo emocional en hemofilia. Madrid: Real Fundación Victoria Eugenia, 1996.

22. Costa M y López E. Educación para la salud. Una estratega para cambiar los estilos de vida. Madrid: Pirámide; 1996.

23. Bayés $R$, Arranz $P$, Barbero J y Barreto P. Propuesta de un modelo integral para una intervención terapéutica paliativa. Med Pal. 1996; 3: 114-121.

24. Lazarus RS \& Folkman S. Stress, Appraisal and Coping. Nueva York: Springer Publishing; 1984.

25. Remor, E. \& The Hemofilia-QoL Group (2004). Desarrollo en España de un nuevo cuestionario de calidad de vida especifico para adultos con hemofilia: el Hemofilia-QoL. Haematológica (ed esp.) 89(3):16-21.

26. García-Llana H, Remor $E$, Selgas R. Adherence to treatment, emotional state and quality of life in patients with end-stage renal disease undergoing dialysis. Psicothema, 2013; (25), Num. 1: 79-8. 